\title{
Rapporto tra salute del cavo orale e dieta nel paziente con patologia renale
}

\author{
M. Bolgiani
}

Dietista - Milano

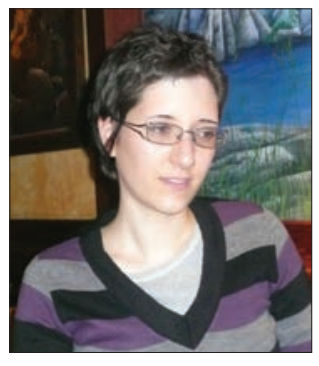

\section{Introduzione}

Nel corso degli anni gli obiettivi della cura nellinsufficienza renale cronica e della dialisi si sono evoluti. Adesso sopravvivere non basta, ma si deve migliorare la qualità di vita e prevenire le disabilità del paziente. Per questi obiettivi, elemento centrale è un corretto stato di nutrizione, inteso sia come composizione corporea che come apporto di nutrienti. Quindi si comprende bene come la salute del cavo orale sia un punto importante, anche se spesso trascurato, della cura dei pazienti con CKD (Chronic Kidney Disease - Malattia Renale Cronica).

Pochi studi hanno esaminato lo stato di salute dei denti e del parodonto nei pazienti con CKD, ma esistono dimostrazioni che esso peggiora con l'inizio della dialisi e quindi, soprattutto in questi pazienti, la salute del cavo orale è un punto di notevole rilevanza (1-4).

Infatti una cattiva salute orale ha inevitabili riflessi negativi sulle condizioni cliniche: può ostacolare un'adeguata alimentazione contribuendo allo sviluppo o alla progressione della malnutrizione, e può favorire l'insorgenza e il mantenimento di focolai di infiammazione cronica (5), anch'essa punto critico del paziente con CKD.

\section{Cavo orale e patologia renale}

Con il progressivo aggravamento dell'insufficienza renale, compaiono segni e sintomi clinici che coinvolgono vari organi e apparati: tra questi non ne è esente il cavo orale che mostra alterazioni morfo-funzionali fino al 90\% nei pazienti con CKD. Queste avranno un ovvio impatto negativo sull'alimentazione del paziente e favoriscono uno stato pro-infiammatorio, con maggior rischio di sviluppo e progressione dell'aterosclerosi e delle patologie cardiovascolari (6). La prevalenza e la severità delle pa- tologie del cavo orale risultano più marcate nei pazienti in terapia emodialitica rispetto ai pazienti in dialisi peritoneale $o$ in terapia conservativa (6). Le patologie del cavo orale descritte nei pazienti con insufficienza renale cronica sono riportate nella Tabella I.

Le parodontopatie, favorite anche dalla scarsa igiene orale dei pazienti, sono tra i fattori causali di uno stato di infiammazione cronica. La placca dentale funge da serbatoio di microrganismi che sono una potenziale causa di batteriemia: ad esempio l'Helicobacter pylori può essere causa di gastrite e di ulcera gastro-duodenale $(7,8)$. Quindi è possibile ipotizzare che le frequenti recidive, che seguono un efficace trattamento eradicante, possano essere dovute al fatto che a questo non si associa anche la bonifica dentale.

La scarsa attenzione per la cura del cavo orale da parte

\section{TABELLA I - PRINCIPALI ALTERAZIONI DEL CAVO ORALE IN PAZIENTI CON IRC}

\author{
Patologie del cavo orale in pazienti con CKD \\ Xerostomia con alterazione del flusso salivare e del $\mathrm{pH}$ salivare \\ Aumento del rischio di sviluppare carie \\ Alitosi e alterazione del gusto \\ Aumento della formazione di tartaro e placca \\ Alterazioni gengivali (sanguinamento, ipertrofia) \\ Aumento della colonizzazione e crescita batterica e quindi di infezioni \\ Parodontopatie \\ Erosione dentale \\ Stomatite uremica, macule e noduli \\ Lesioni precancerose (leucoplachia) \\ Lesioni cancerose (carcinoma a cellule squamose e sarcoma) \\ Lesioni dell'osso alveolare \\ Ritardo dell'eruzione dei denti
}




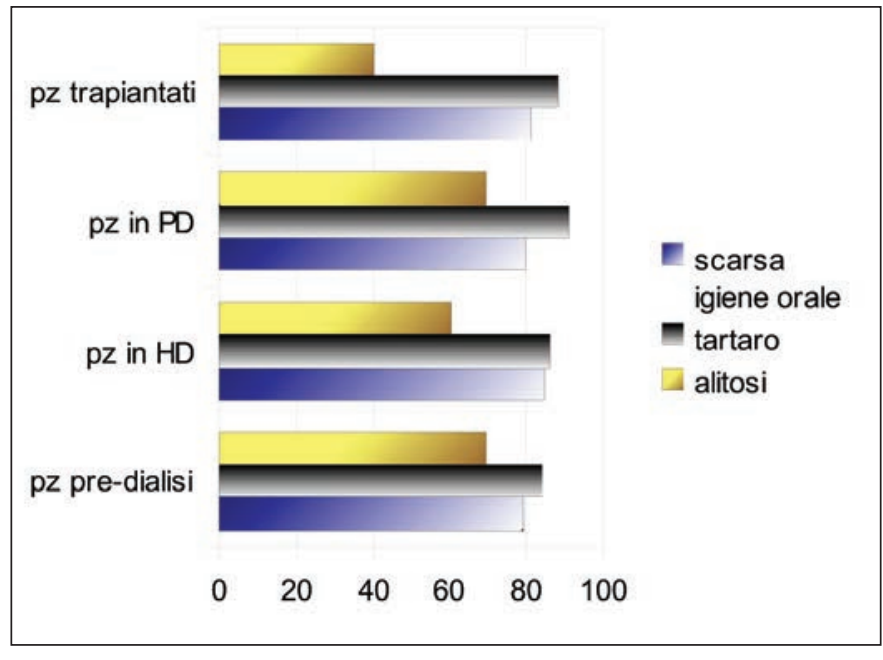

Fig. 1 - Prevalenza nei pazienti con patologia renale di cattiva igiene orale, tartaro e alitosi.

Modificato da Souza CM, et al. Oral health in Brazilian patient with chronic renal disease. Rev Med Chile 2008; 136: 741- 746.

dei pazienti può essere legata all'età spesso avanzata, alle implicazioni psicologiche indotte dalla dialisi, allo stress, ansietà, depressione, alla scarsa consapevolezza sull'importanza dell'igiene orale o al possibile rifiuto di misure preventive e cure da parte degli stessi pazienti. Souza et al (9) hanno condotto uno studio su 286 pazienti brasiliani di cui 13 in pre-dialisi, 158 in emodialisi, 23 in dialisi peritoneale e 92 trapiantati di rene (Fig. 1). Gli Autori hanno rilevato cattive condizioni del cavo orale nell'83\% della popolazione studiata, con un'alta prevalenza di tartaro dentale (87\%) nei pazienti in emodialisi e soprattutto in quei pazienti che utilizzano calcio carbonato e hanno una scarsa igiene orale. L'alitosi è stata riscontrata nel $55 \%$ della popolazione studiata. Nei pazienti in dialisi sono stati osservati anche la riduzione della secrezione salivare accompagnata da atrofia e fibrosi delle ghiandole salivari, spesso asintomatica (10), e un prevalente sviluppo di organismi urea-litici con la conseguente formazione di ammonio (e alitosi).

Nella CKD vi è anche un'alta prevalenza della carie dentale che, come riferiscono Souza et al, risulta più bassa nei pazienti in emodialisi per l'effetto antibatterico dell'urea e per l'aumento del tartaro: l'indice DMFT per tutta la popolazione con CKD è risultato essere 20,6 rispetto al DMFT per la popolazione generale sana di 13,9 (11) (Fig. 2). La prevalenza e la gravità delle carie sono espresse con l'indice DMFT/dmft, introdotto da Klein e Palmer nel 1938, che rappresenta il numero medio dei denti (Teeth), di quelli cariati (Decayed), mancanti per

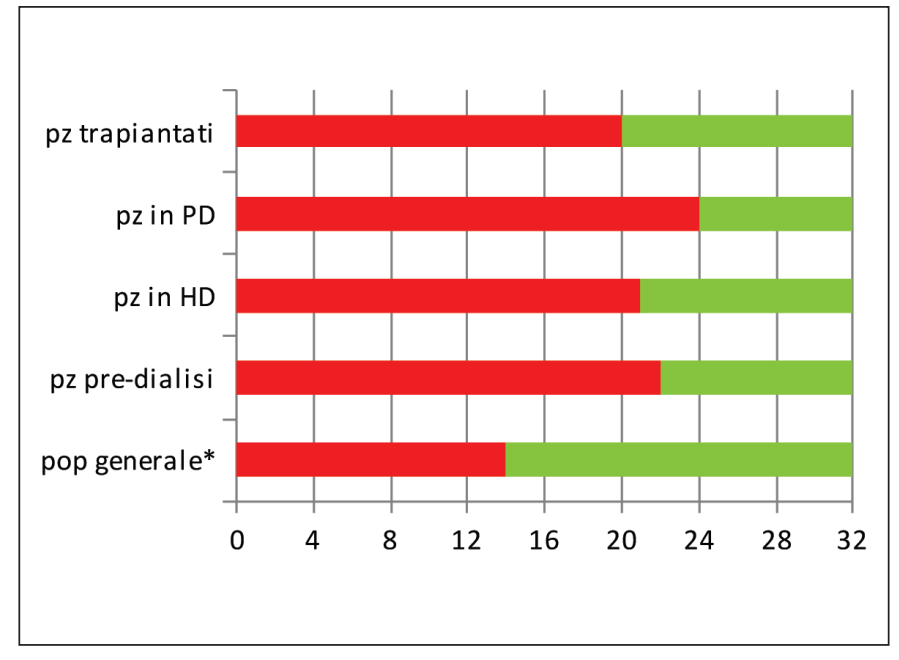

Fig. 2 - L'indice DMFT nei pazienti con patologia renale. Modificato da Souza CM, et al. Oral health in Brazilian patient with chronic renal disease. Rev Med Chile 2008; 136: 741-746. ${ }^{*}$ Peterson PE, et al. The global burden of oral diseases and risks to oral health. Bulletin of World Health Organization 2005: 83 (9).

carie (Missed) o otturati per carie (Filled): il maiuscolo si riferisce ai denti permanenti, il minuscolo ai denti decidui. L'OMS consiglia una valutazione del suddetto indice a 5 anni per i denti decidui, a 12, 15, 35-44, 65-74 anni per i denti permanenti.

Il trattamento delle patologie orali nei pazienti con CKD è abbastanza complesso sia per le comorbidità presenti, come il diabete e l'immunodepressione, sia per i farmaci utilizzati come gli anticoagulanti, gli antiaggreganti e gli antipertensivi.

I pazienti trapiantati hanno una minore incidenza di alitosi, ma una maggiore incidenza di ipertrofia gengivale indotta dagli immunodepressori (come la ciclosporina) a cui si collega un più alto rischio di infiammazione gengivale e di formazione della placca dentale (9).

Lo studio di Gurkan et al (12) condotto su 145 pazienti ha confermato una scarsa igiene orale quotidiana $(85,7 \%)$ dei pazienti e una presenza sulla superficie dentale di placca e tartaro $(93,7 \%)$ causati da un non corretto spazzolamento e da una non accurata pulizia interdentale.

In questo studio, nessuno dei pazienti è stato indirizzato ad andare da un dentista per la cura del cavo orale.

Le patologie del cavo orale influenzano direttamente l'assunzione di cibo, anche se in modo variabile da soggetto a soggetto.

In linea generale lo sviluppo di parodontopatie, con conseguente dolore e dolorabilità, gonfiore gengivale e perdita di denti, causa difficoltà nella masticazione 
e una maggiore sensibilità dentale; per questo si preferiranno cibi morbidi, non troppo caldi o non troppo freddi.

Invece la presenza di xerostomia e alitosi, con conseguente secchezza delle fauci e sgradevole sapore in bocca, porta a una riduzione dell'introito di alimenti aggravato anche dall'alterazione del gusto, alla repulsione verso alcuni alimenti, specialmente quelli che hanno un sapore e un odore "particolare" come possono essere il pesce o alcuni tipi di verdura e frutta. Inoltre si verificherà un forte impulso a bere di più, soprattutto bevande fredde/ghiaccio, con un maggior rischio di eccessivo incremento ponderale nei pazienti in dialisi. Se interviene anche la nausea vi è repulsione per tutto ciò che è liquido o emana forti aromi per cui si prediligono alimenti "duri, secchi e inodori" come grissini, cracker, fette biscottate ecc.

Il maggior rischio di formazione di carie deve indurre il paziente a modificare la dieta, sotto consiglio del nefrologo/dentista/dietista, dando la preferenza a cibi non cariogeni e a ridurre il tempo di permanenza del substrato nella bocca.

Per tutti questi motivi è importante che anche il dietista valuti lo stato di salute del cavo orale del paziente e dia, di conseguenza, indicazioni e consigli nutrizionali appropriati alle problematiche. $\mathrm{E}$ necessario fare tutto questo come prevenzione e quindi in maniera regolare prima che si sviluppino tali patologie.

Emerge chiaramente il bisogno di una maggiore sensibilizzazione e implementazione di programmi destinati alla cura del cavo orale, che fino a oggi è lasciata alla singola iniziativa personale. Per far questo è necessaria la stretta collaborazione tra nefrologo e dentista (13), e si possono suggerire pochi e semplici consigli per la cura del cavo orale, quali quelli dopo specificati.

I pazienti in trattamento emodialitico o portatori di trapianto devono essere valutati con particolare attenzione. È molto importante bonificare eventuali foci di infezione del cavo orale e considerare una profilassi antibiotica nel caso di interventi che potrebbero portare a sanguinamento o a rischio di batteriemia come estrazione, trattamenti parodontali, ortodontici, chirurgici. Il trattamento dentale dovrebbe essere svolto nei giorni di non dialisi e comunque dopo una valutazione attenta dell'esame emocromocitometrico e della coagulazione. Anche se in letteratura le opinioni sono contrastanti, sarebbe necessario proporre un trattamento antibiotico preventivo con amoxicillina per ridurre il rischio di infezione batterica, e di endocardite. Si consiglia infine di non ricorrere a trattamenti dentali nei primi sei mesi successivi al trapianto.

\section{Consigli generali utili per la cura del cavo orale}

Utilizzare il filo interdentale almeno una volta al giorno. Spazzolare i denti dopo ogni pasto.

Fare attenzione alla modalità di spazzolamento dei denti. Lo spazzolino, con una inclinazione di 45 gradi, va mosso in direzione perpendicolare alla gengiva e sempre dalla gengiva verso il dente, sia sulla parte esterna della dentatura che su quella interna. Nella pulizia della parte masticatoria dei denti posteriori lo spazzolino va mosso, invece, prima dal dietro verso l'avanti e viceversa e poi lateralmente per pulire gli interstizi dentali.

Spazzolare il dorso linguale con appositi strumenti pulisci lingua per eliminare i microrganismi responsabili dell'alitosi.

Utilizzare collutori a base di sostanze ad azione antisettica oppure infusi con erbe naturali facendo degli sciacqui più volte al giorno.

Utilizzare collutori, chewing-gum o pastiglie da sciogliere in bocca integrati con probiotici. Gli studi che fino ad ora sono stati condotti hanno dimostrato un ruolo positivo dei probiotici sull'ambiente microbiologico orale come ad esempio una riduzione della placca, dell'infiammazione gengivale, delle carie e dell'alitosi (14-19).

Provvedere alla cura di eventuali patologie dentali e/o gengivali.

In conclusione, la patologia e la cura del cavo orale sono argomenti spesso trascurati nei pazienti affetti da malattia renale cronica. Un maggior rischio di infezioni, di infiammazione cronica e di malnutrizione rappresentano le principali conseguenze cliniche, tutte di particolare rilevanza nei pazienti nefropatici cronici.

Una maggiore consapevolezza del problema da parte dei nefrologi e dei pazienti e lo sviluppo di programmi di educazione, di screening, di prevenzione e di cura delle patologie del cavo orale, possono aumentare la qualità di cura e con esse la qualità di vita dei pazienti nefropatici cronici.

\section{Riassunto}

L'igiene della bocca e la diagnosi e trattamento delle patologie del cavo orale sono aspetti spesso trascurati nei pazienti affetti da malattia renale cronica. Un maggior rischio di infezioni, di infiammazione cronica e di malnutrizione rappresentano le principali conseguenze cliniche, tutte di particolare rilevanza nei pazienti nefropatici cronici, di una cattiva gestione della salute del cavo orale. Una maggiore consapevolezza del problema da parte dei nefrologi/dentisti/dietisti e dei pazienti e lo sviluppo di programmi di educazione, di screening, 
di prevenzione e di cura delle patologie del cavo orale, possono contribuire ad elevare la qualità della cura dei nefropatici cronici.

Parole chiave: Malattia renale cronica, Cavo orale, Malnutrizione, Parodontopatie

\section{Indirizzo degli Autori:}

Dr. Monica Bolgiani

Dietista

Via Medea 18

20138 Milano

monicabolgiani@hotmail.it

\section{Bibliografia}

1. Thorman R, Neovius M, Hylander B. Clinical findings in oral health during progression of chronic kidney disease to end-stage renal disease in a Swedish population. Scand J Urol Nephrol 2009; 43(2): 154-9.

2. Chamani G, Zarei MR, Radvar M, Rashidfarrokhi F, Razazpour F. Oral health status of dialysis patients based on their renal dialysis history in Kerman, Iran. Oral Health Prev Dent 2009; 7 (3): 269-75.

3. Bayraktar G, Kurtulus I, Duraduryan A, et al. Dental and periodontal findings in hemodialysis patients. Oral Dis 2007; 13(4): 393-7.

4. Cengiz MI, Sümer P, Cengiz S, Yavuz U. The effect of the duration of the dialysis inhemodialysis patients on dental and periodontal findings. Oral Dis 2009; 15(5): 336-41.

5. Bayraktar G, Kurtulus I, Kazancioglu R, et al. Oral health and inflammation in patients with end-stage renal failure. Perit Dial Int 2009; 29: 472-9.

6. Borawski J, Wilczynska-Borawska M, Stokowska W, Mysliwiec M. The periodontal status of pre-dialysis chronic kidney disease and maintenance dialysis patients.
Nephrol Dial Transplant 2007; 22: 457-64.

7. Al-Hawajri AA, Keret D, Simhon A, et al. Helicobacter pylori DNA in dental plaques, gastroscopy, and dental devices. Dig Dis Sci 2004; 7/8: 1091-4.

8. Anand PS, Nandakumar K, Shenoy KT. Are dental plaque, poor oral hygiene and periodonatl disease associated with Helicobacter pylori infection? J Periodontol 2006; 77: 692-8.

9. Souza CM, Braosi APR, Luczyszyn SM, et al. Oral health in Brazilian patient with chronic renal disease. Rev Med Chile 2008; 136: 741-6.

10. Pastorino M, Catalano C, Martorano C, et al. Salivary and lacrimal secretion is reduced in patients with ESRD. Am J Kidney Dis 2003; 42: 722-8.

11. Peterson PE, et al. The global burden of oral diseases and risks to oral health. Bulletin of World Health Organization; September 2005: 83 (9).

12. Gurkan A, Kose T, Atilla G. Oral health status and oral hygiene habits of an adult turkian population on dialysis. Oral Health Prev Dent 2008; 6: 37-43.

13. Cerverò AJ, Bagàn JV, Soriano YJ, Roda PR. Dental management in renal failure: patients on dialysis. Med Oral Patol Oral Cir Bucal 2008; 13: E419-26.

14. Teughels W, Loozen G, Quirynen M. Do probiotics offer opportunities to manipulate the periodontal oral microbiota? J Clin Periodontol 2011; 38(Suppl. 11): S159-77.

15. Harini PM, Anegundi RT. Efficacy of a probiotic and chlorhexidine mouth rinses: A short-term clinical study. J Indian Soc Pedod Prev Dent 2010; 28: 179-82.

16. Saraf K, Shashikanth MC, Priy T, Sultana N, Chaitanya NC. Probiotics do they have a role in medicine and dentistry? J Assoc Physicians India 2010; 58: 488-90, 495-6.

17. Sinkiewicz G, Cronholm S, Ljunggren L, Dahlén G. Influence of dietary supplementation with Lactobacillus reuteri on the oral flora of healthy subjects. Swed Dent J 2010; 34(4): 197-206.

18. Vivekananda MR, Vandana KL, Bhat KG. Effect of the probiotic Lactobacilli reuteri (Prodentis) in the management of periodontal disease: a preliminary randomized clinical trial. J Oral Microbiol 2010; doi: 10.3402/jom. v2i0.5344.

19. Bonifait L, Chandad F, Grenier D. Probiotics for oral health: myth or reality? J Can Dent Assoc 2009; 75(8): 585-90. 